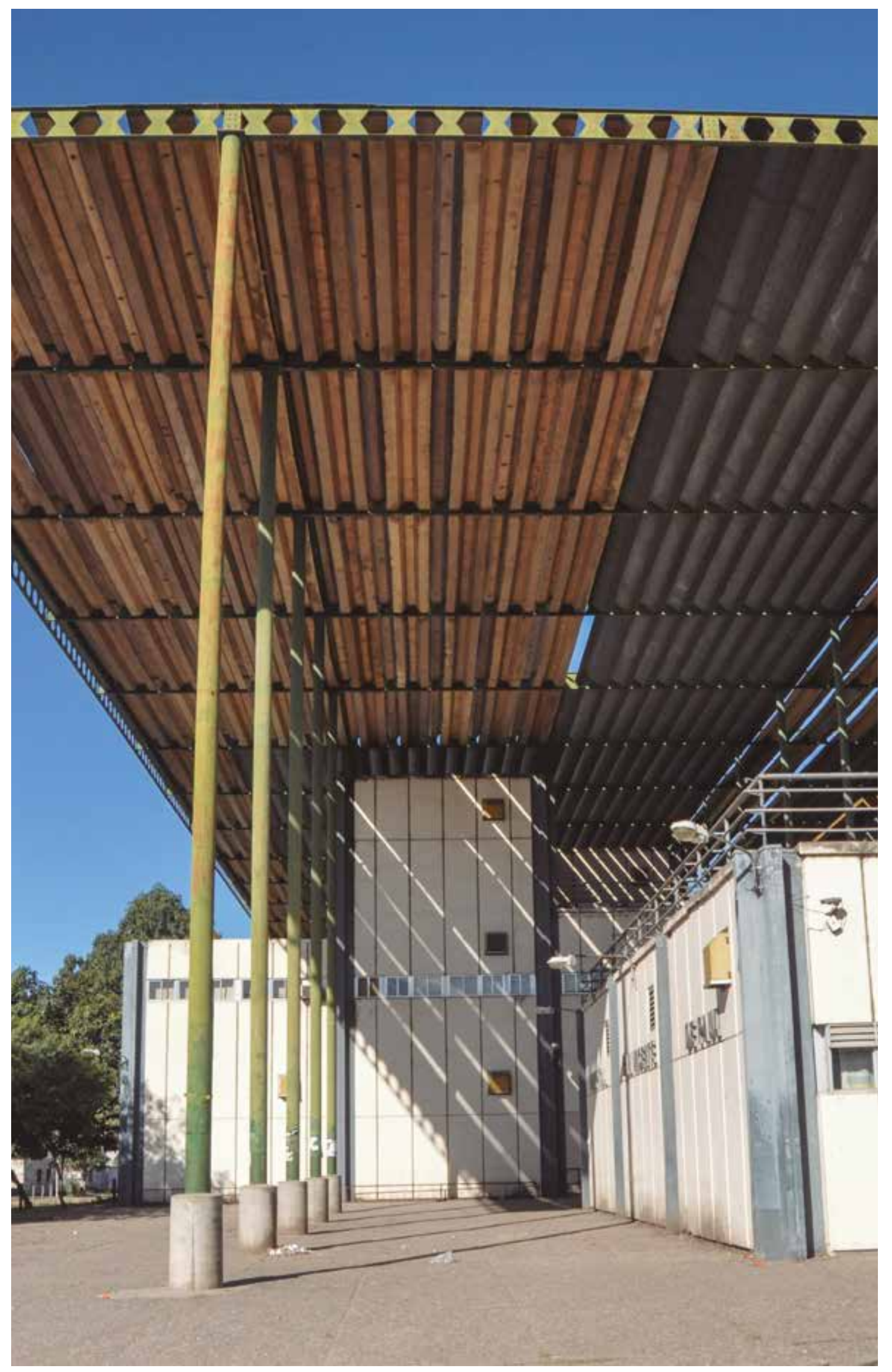

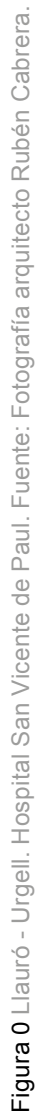



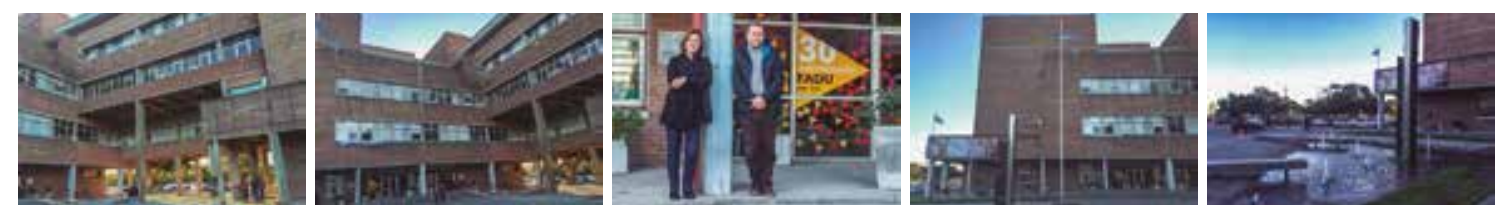

\title{
ARQUITECTURA DE SISTEMAS Y PROGRAMAS SOCIALES EN LA ARGENTINA DESARROLLISTA: DEL OPTIMISMO A LO POSIBLE'
}

\author{
SYSTEMS ARCHITECTURE AND SOCIAL PROGRAMS IN DEVELOPMENTALIST \\ ARGENTINA: FROM OPTIMISM TO THAT WHICH IS POSSIBLE'
}

Luis Müller², Cecilia Parera ${ }^{3}$

RESUMEN

El contexto de consolidación del modelo estatal desarrollista en Argentina, desplegado desde la segunda posguerra, impulsó la industria como vía de mejora económica, mientras la arquitectura se transformó en un actor central en la intención de brindar herramientas válidas para satisfacer distintas necesidades básicas. En materia de salud, los concursos de anteproyectos para hospitales nacionales de fines de la década de 1960 constituyeron un fecundo campo de exploración proyectual y tecnológica, y brindaron una oportunidad para la difusión de la llamada "arquitectura de sistemas" como alternativa a los métodos vigentes. Este artículo toma como caso de análisis el proyecto ganador para el Hospital San Vicente de Paul en Orán, Salta, identificando en la flexibilidad, la indeterminación y el crecimiento, así como la incorporación de sistemas constructivos industrializados, las herramientas proyectuales específicamente elegidas por sus autores como alternativa para mejorar la calidad de vida de esta población postergada.

Palabras clave: arquitectura, sistemas, Argentina, hospitales, industrialización.

ABSTRACT

The consolidation of the developmentalist government model in Argentina deployed after the Second World War impelled industry as the path to economic improvement, while architecture became a central player in the attempt to provide valid tools to meet different basic needs. In the area of healthcare, at the end of the decade of the 1960's, bids for the preliminary design of national hospitals became fertile ground for design and technological exploration and presented an opportunity to disseminate so-called "systems architecture" as an alternative to existing methods. The case analyzed for this article is the winning project for the San Vicente de Paul Hospital in Orán, Salta. Its flexibility, indeterminacy and growth, as well as the incorporation of industrialized building systems, demonstrate the design tools specifically chosen by its authors as alternatives to improve the quality of life of this neglected population.

Keywords: architecture, systems, Argentina, hospitals, industrialization.

Articulo recibido el 18 de marzo de 2016 y aceptado el 8 de junio de 2016

[1] Este artículo forma parte de las reflexiones desarrolladas en el marco de dos proyectos de investigación: C.A.I. + D. '2011: "Hacia una arquitectura de sistemas. Proyectos en Argentina (1948-1971)". (Dirección: Mg. Arq. Luis Müller. Facultad de Arquitectura, Diseño y Urbanismo. Universidad Nacional del Litoral, Argentina. 2013 - 2016); y PICT 2013-1486 ANPCYT "Arquitectura, tecnología y proyecto: obras públicas e infraestructura urbana y territorial en Argentina (1955-1971)". (Dirección: Mg. Arq. Luis Müller. FADU, UNL. 2014 2016).

[2] Profesor e investigador INTHUAR, Facultad de Arquitectura, Diseño y Urbanismo. Universidad Nacional del Litoral. Santa Fe,

Argentina. Imuller@fadu.unl.edu.ar

[3] Profesora e investigadora INTHUAR, Facultad de Arquitectura, Diseño y Urbanismo. Universidad Nacional del Litoral. Santa Fe, Argentina.cparera@fadu.unl.edu.ar 
En el contexto económico-productivo de la segunda posguerra y la tensa polarización militar definida por la Guerra Fría, las teorías económicas clásicas fueron cuestionadas ante el reconocimiento del marcado deterioro en los términos de intercambio, que actuaba en perjuicio de aquellas economías primario-exportadoras. En este período, en Latinoamérica se manifestaron diversas interpretaciones, particularmente en el marco de la CEPAL ${ }^{4}$, como la dualidad "centro/periferia" y la "Teoría de la dependencia", que identificaban que las características sociopolíticas propias de la región tendían a obstaculizar su desarrollo económico. Estas respuestas teóricas se transformaron en los pilares conceptuales de un nuevo modelo estatal, el "Desarrollismo", que se fundó en el impulso de políticas de industrialización por sustitución de importaciones a fin de reducir la dependencia del mercado interno respecto de bienes provenientes de otros países. La progresiva "asimilación de la técnica moderna" mejoraría el rendimiento y la racionalización de la estructura productiva de los países "en desarrollo" (Prebisch, 1952). De esta manera, el Estado no era más "benefactor" o "asistencialista", como en términos generales se proponía desde la crisis económica de 1930, sino que asumía el rol de controlador de las políticas de inversión, definiendo las áreas económicas que debían ser fomentadas (Rapoport, 2005).

[4] Comisión Económica para América Latina (CEPAL), organismo creado por la Organización de las Naciones Unidas en 1947. En particular, las diversas publicaciones de este organismo así como los escritos de Raúl Prebisch, Theotonio dos Santos y Celso Furtado, entre otros, constituyen referencias insoslayables de este ideario. 
En la década de 1950 diversos gobiernos latinoamericanos asumieron estos lineamientos, habilitando el ingreso de capital extranjero para consolidar las industrias básicas, como la petrolera, la química, la metalúrgica y la maquinaria, y creando una serie de empresas estratégicas para lograr el equilibrio del desarrollo nacional. Las gestiones de Adolfo Ruiz Cortines en México (período 1952/1958), Carlos Ibáñez del Campo en Chile (1952/1958), Juscelino Kubitschek en Brasil (1956/1961) y Arturo Frondizi en Argentina (1958/1962), son testimonio del referido modelo. La opinión de Rogelio Frigerio, Secretario de Relaciones Socioeconómicas en los inicios de la mencionada presidencia argentina, es elocuente de este ideario, posicionando al reequilibrio regional y a la incorporación de tecnología como las claves para el éxito del modelo:

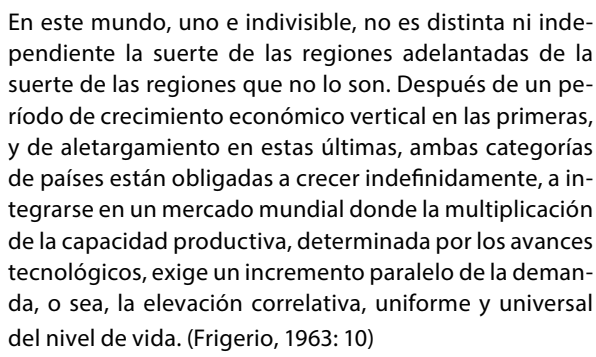

En este mundo, uno e indivisible, no es distinta ni independiente la suerte de las regiones adelantadas de la suerte de las regiones que no lo son. Después de un período de crecimiento económico vertical en las primeras, y de aletargamiento en estas últimas, ambas categorías de países están obligadas a crecer indefinidamente, a integrarse en un mercado mundial donde la multiplicación de la capacidad productiva, determinada por los avances tecnológicos, exige un incremento paralelo de la demanda, o sea, la elevación correlativa, uniforme y universal del nivel de vida. (Frigerio, 1963: 10)

Si bien la inestabilidad política signó la década de 1960 en Argentina, con la destitución de los Presidentes Constitucionales, Arturo Frondizi (período 1958/1962) en 1962 y Arturo Illia (1963/1966) en 1966, esa voluntad por superar el desarrollo desigual mantuvo su vigencia, principalmente en materia de desarrollo tecnológico y provisión de servicios básicos. Diversas herramientas de planificación concebidas por sus antecesores fueron sostenidas por los gobiernos de facto, como la Comisión Nacional para el Desarrollo -CONADE, creada por el gobierno de Frondizi en 1961-, atendiendo sobre todo a sus análisis en materia de vivienda, educación, salud y transporte. En lo que refiere a la problemática sanitaria, de interés para el presente artículo, por estos años se verifica una sustancial modificación de las estructuras de gobierno, con la creación del Ministerio de Bienestar Social en $1966^{5}$ y del Programa Nacional de Planeamiento Físico y Arquitectura en 1968, dependiente de la Subsecretaría de Salud Pública del mencionado Ministerio. El estudio de la red de establecimientos de salud a nivel regional realizado por el equipo técnico del referido programa, que incluía el análisis de la capacidad hospitalaria instalada, de la tecnología disponible, de su accesibilidad y de la población beneficiada, resolvió "el establecimiento de un sistema que [...] [asegurara] la atención médica de todas las personas, cubriendo todos los aspectos de la prestación y encontrando como particular destinatario a la población rural y urbana de menores recursos" (Subsecretaría de Salud Pública, 1971: 29). En concreto, el estudio tomó como caso piloto, para realizar un ensayo de la metodología elaborada, a la región del Noroeste Argentino, la que por esos años alojaba el $10 \%$ de la población del país y constituía el
$15 \%$ de la superficie nacional. El trabajo de campo identificó significativos niveles de desigualdad, así como la necesidad de construir unidades de atención médica de alta "complejidad", entendiendo este concepto como un indicador que precisaba "el número de tareas diversificadas que [...] [integraban] la actividad global de un establecimiento y el grado de desarrollo alcanzadas por las mismas" (ibídem: 35).

Este artículo se propone indagar en las experiencias proyectuales desarrolladas en el marco de estos análisis, a fin de dar cuenta de las alternativas que asumieron a la arquitectura como una vía posible de mejora de la calidad de vida de la población. En esta línea, estas experiencias son estudiadas como productos de la cultura vinculadas a los procesos sociopolíticos y económicos, pero que no pierden su especificidad dentro del campo de la producción y del debate de ideas de la arquitectura. Es por ello que se desarrolla aquí un análisis de lógica cualitativa, en el que las explicaciones interpretativas constituyen la base de la comprensión.

\section{LA ARQUITECTURA DE SISTEMAS, UNA RESPUESTA POSIBLE}

La convocatoria a ocho Concursos de Croquis Preliminares impulsada por el Ministerio de Bienestar Social en 1969 y con el auspicio de la Sociedad Central de Arquitectos activó una ágil y dinámica manera de obtener variadas propuestas de anteproyectos (Liernur, 2001). Dada su complejidad, en gran parte de los casos, al momento de concretar la obra, los equipos ganadores continuaban vinculados para desarrollar el proyecto final con apoyo de las oficinas técnicas estatales y de consultores especializados. Por otro lado, esta modalidad de selección posibilitó la emergencia de equipos constituidos por jóvenes arquitectos y la consolidación de nuevas líneas proyectuales. Resulta significativo que la voluntad de generar una arquitectura que sintonizara con las más innovadoras tendencias que se manifestaban en el mundo, ya estuviera presente en las mismas "Pautas de diseño" elaboradas por la repartición estatal convocante. Sin embargo, esto no fue casual, sino el resultado de un actualizado estudio que llevó a cabo un equipo constituido en 1968 por la Subsecretaría de Estado de Salud Pública del Ministerio de Bienestar Social, con el objetivo de proponer criterios para el diseño de las nuevas infraestructuras hospitalarias. Entre las recomendaciones emitidas, podía leerse:

\footnotetext{
Las últimas investigaciones establecen pautas de "indeterminación" para el diseño de unidades de atención médica. La "indeterminación" es debida, fundamentalmente, a los procesos de "cambio" y a las necesidades de "crecimiento". [...]
}

La adopción de proyectos arquitectónicos que contemplen dichas pautas en su diseño: flexibilidad, adecuación 
de locales a usos distintos, previsiones en el aumento o disminución de los servicios, y la resolución de las instalaciones complementarias como una malla que permita diferentes alternativas de conexión y fácil acceso a su recorrido para su mantenimiento, redundará en beneficio del mantenimiento del establecimiento. [...]

Es decir que las soluciones arquitectónicas deberán proponer un "sistema" espacial que integre:

- La estructura resistente como una malla tridimensional.

- Una red de instalaciones que asegure la provisión de los servicios a cualquier punto del establecimiento.

- Una trama circulatoria que garantice los traslados de elementos y personas, convenientemente diferenciados.

- Espacios flexibles que permitan su adecuación a diferentes posibilidades de uso y expansión. [...

En el momento actual, en los países más adelantados, los hospitales "indeterminados" se construyen recurriendo a los más modernos sistemas de ejecución, aplicando las técnicas de prefabricación integral. En nuestro país se tiende a esta meta y se trata de aplicar los medios de ejecución más avanzados entre nosotros.

(Ministerio de Bienestar Social, 1971:30)

Estos lineamientos asumían claramente la incorporación de innovaciones tecnológicas, tanto en materia de equipamiento médico como técnicas constructivas, y se condecían con la tendencia sistémica en expansión en el debate disciplinar contemporáneo, sobre el que sin duda los profesionales de la mencionada repartición estatal estaban informados. La noción de "sistema", tomada del campo de la biología, entendía a los organismos como conjuntos con propiedades específicas que no podían ser reducidas a las partes de sus componentes, y en los que las relaciones entre ellas asumían un rol preponderante (Von Bertalanffy, 1968). En la década de 1950, la "Teoría General de los Sistemas" alcanzó las más diversas aplicaciones, intentando dar una respuesta a la creciente complejidad que afectaba al mundo industrializado. En el campo de la arquitectura, la necesidad de reconstrucción de las ciudades europeas afectadas por los bombardeos, las estrategias de reconversión de la industria bélica aplicada a la renovación edilicia, y la amplia disponibilidad de nueva tecnología y sistemas constructivos, entre otros factores, impulsaron un cambio de paradigma proyectual, intentando "clarificar el proceso de diseño, descomponer las partes del programa, poder visualizar científicamente sus propiedades para luego poder re-ensamblarlas con certeza" (Aliata 2014: 15). La renovación es evidente en producciones de diversa naturaleza; en el proyecto para el Hospital de Venecia de Le Corbusier (1962/1965) (ver Figura 1), en el conjunto edilicio de la Universidad Libre de Berlín de Candilis, Josic, Woods y Schiedhelm (1963-1973), en los escritos de Richard Llewelyn-Davies y Hugh Macaulay (1966), y en las especulaciones metodológicas de varios miembros del Team X -principalmente Cedric Price y Aldo Van Eyck- $y$ del Metabolismo Japonés - de la mano de Kenzo Tange y Kisho Kurokawa-, entre otros, o las derivaciones que llevarían a Alison Smithson a proponer en 1974 el concepto de "mat-building" como un factor común que identificaba a gran parte de esta arquitectura.

Como agudamente identificara por esos años el arquitecto y crítico argentino Francisco Bullrich (1969), la "arquitectura de sistemas" se caracterizaba por una organización espacial de crecimiento indefinido en base de una agregación celular, una forma abierta para agregar o quitar elementos de acuerdo con requerimientos variables, la previsión de la obsolescencia y de la flexibilidad para hacer frente al crecimiento por etapas o a variaciones, así como el uso de sistemas constructivos industrializados. Esta modalidad de proyecto alcanzó gran difusión en Argentina a partir de diversas publicaciones, como Summa, Cuadernos Summa Nueva Visión, Trama, y la Revista de la Sociedad Central de Arquitectos y Ambiente, y encontró en la variedad de concursos concretados entre fines de la década de 1960 y principios de la siguiente, el ámbito adecuado para la exploración sobre el proyecto, tal como lo demuestran las propuestas ganadoras para la embajada Argentina en Brasilia en 1970 -primer premio otorgado al arquitecto Francisco Bullrich-, el Centro Cívico para la Provincia de San Juan

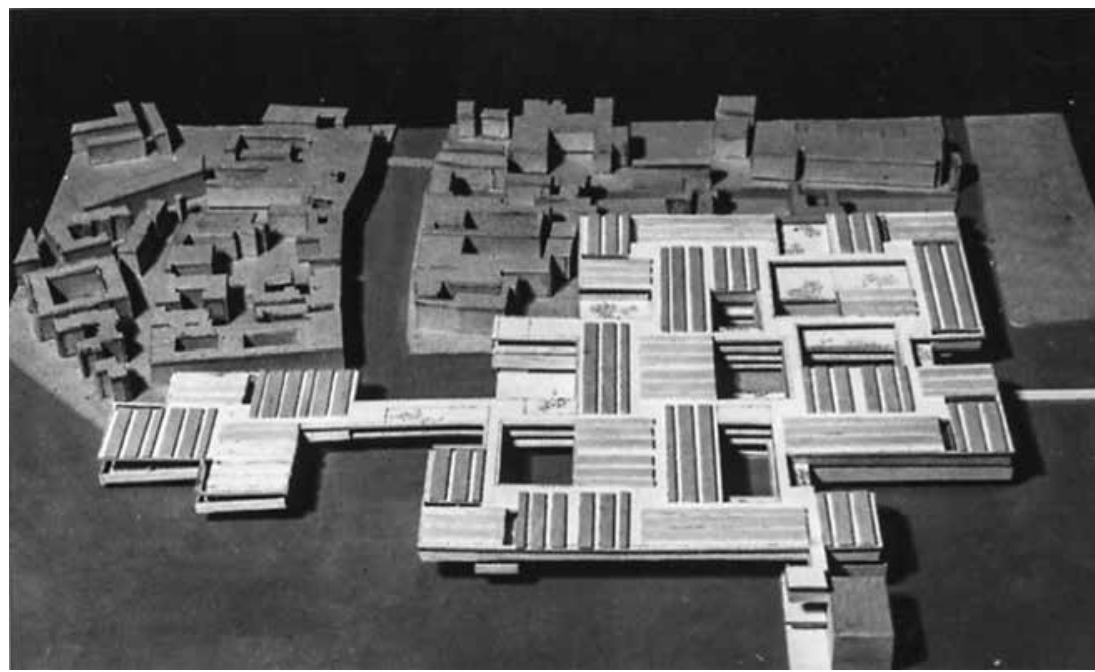

Figura 1 Le Corbusier. Maqueta del Hospital de Venecia (1965). Fuente: Fundación Le Corbusier. 
en 1971 -estudio Llauró y Urgell y Asociados- y los concursos ganados -aunque no construidos- por el estudio Baudizzone, Díaz, Erbin, Lestard, Varas para la Facultad de Ciencias Exactas y Naturales de la Universidad Nacional de La Plata en 1968 y el Auditorio de Buenos Aires en 1972. Más aún, el campo profesional local también se encontraba profundamente abocado al estudio de la renovación que se verificaba, como demuestran trabajos contemporáneos de los arquitectos Arturo Montagú (1972), Raúl Rivarola (1971) y Mario Gandelsonas (1970), entre otros, o a la decidida puesta en acción de la "teoría de los sistemas", como haría pocos años después Fermín Estrella (1985) con la aplicación de su interpretación de los sistemas a la arquitectura escolar y a la producción de la vivienda popular. A partir de estas reflexiones es posible reconocer la estrecha relación que se planteaba entre nuevos sistemas de proyecto y nuevos sistemas constructivos $^{6}$

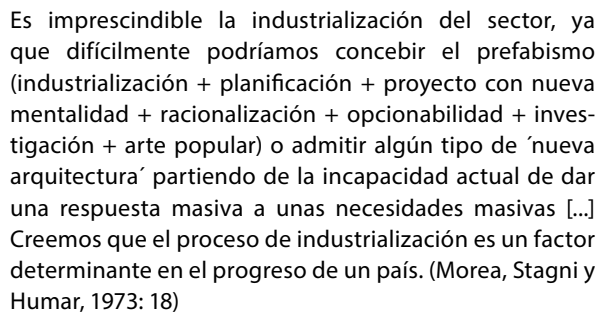

Es imprescindible la industrialización del sector, ya que difícilmente podríamos concebir el prefabismo (industrialización + planificación + proyecto con nueva mentalidad + racionalización + opcionabilidad + investigación + arte popular) o admitir algún tipo de 'nueva arquitectura' partiendo de la incapacidad actual de dar una respuesta masiva a unas necesidades masivas [... Creemos que el proceso de industrialización es un factor determinante en el progreso de un país. (Morea, Stagni y Humar, 1973: 18)

El reconocimiento del rol decisivo que la tecnología había alcanzado para la disciplina de la arquitectura no era una novedad; más bien, había sido anticipado por Siegfried Giedion en Mechanization takes command, a contribution to anonymous history (1948), había constituido el sustento para impulsar la revisión historiográfica del Movimiento Moderno -reforzado por Reyner Banham en Theory and design in the first machine age (1960)-, y había sido llevado a extremos que, por entonces, rozaban la ciencia ficción en sus expectativas. Los proyectos de Cedric Price, Yona Friedman, Archigram y muchos otros ilustraban esta década en que la "utopía tecnológica", basada en la idea moderna de un progreso ilimitado, mantenía su vigencia propugnando por el aumento ilimitado del consumo de energía y el máximo aprovechamiento de los avances científicos (Shmidt y Müller, 2013).

\section{EL PROYECTO GANADOR PARA EL HOSPITAL DE ORÁN UNA PROPUESTA SISTÉMICA}

De los ocho concursos para hospitales en Argentina que fueron mencionados sólo se construyeron dos, el Hospital Nacional de Pediatría en la ciudad de Buenos Aires y el Hospital San Vicente de Paul en la localidad de Orán. Mientras que el primero debía atender a la población infantil de la capital del país y su zona de influencia, el segundo debía concentrarse en la atención de todas las enfermedades que afectaban a los pacientes ambulatorios de la zona rural del Chaco Salteño, así como ocuparse de las campañas de educación sanitaria de su población. Las bases de este último concurso, elaboradas en forma conjunta entre la Dirección de Vivienda y Arquitectura de la Provincia de Salta, la Subsecretaría de Salud Pública de la Nación y la Sociedad Central de Arquitectos, estaban en un todo de acuerdo con las "Pautas de Diseño" desarrolladas por el Ministerio de Bienestar Social de la Nación, y virtualmente conducían el juicio de los jurados hacia un tipo de proyecto enrolado en las corrientes sistémicas. El primer premio fue otorgado a la propuesta presentada por el Estudio Llauró - Urgell y Asociados, en cuya memoria descriptiva sus autores anunciaban su adhesión a la referida estrategia proyectual: "El interés principal de nuestro estudio en la etapa de proyecto fue el desarrollo de una arquitectura de sistemas que permitiera el crecimiento y el cambio de destino de los locales manteniendo coherencia y unidad, sin la limitación de esquemas formales rígidos, impuestos de antemano" (Schere, 2008: 340).

La organización abierta, sistemática y posible de expandirse en cualquier sentido en una planta de perímetro libre, en una deliberada indeterminación volumétrica subordinada a una trama modular, resulta lógica en el proyecto, no sólo por encontrarse plenamente consustanciada con las teorías arquitectónicas de su tiempo, sino porque prácticamente se hacía cargo de las solicitaciones establecidas en las directivas del concurso. Como es posible apreciar en la Figura 2, el programa se ordena en el predio disponiendo áreas funcionales que ocupan casi la totalidad de la superficie disponible en planta baja, y que se articulan en torno al vestíbulo de entrada, referencia formal y funcional que orienta a los pacientes. La planta alta tiene menor densidad de ocupación, generando terrazas y evitando la percepción de monotonía que la marcada horizontalidad del conjunto hubiera podido definir a las fachadas.

Reconociendo la necesidad de un mantenimiento permanente y una constante renovación de los equipamientos e instalaciones médicas, se dispuso de entrepisos técnicos que aseguraran la accesibilidad en todo el edificio y la posibilidad de introducir cambios sin interferir en el funcionamiento normal de los servicios. El planteamiento técnico-constructivo del proyecto apelaba a nociones de gran flexibilidad, para lo que el sistema constructivo fue pensado como un enorme juego de meccano con piezas prefabricadas de hormigón armado para ensamblar?, desmontar y volver a armar, en un patrón capaz de incorporar más unidades repetibles a medida que así lo planteasen las necesidades del hospital. La totalidad del proyecto fue modulado en múltiplos de 0,56 metros, en atención a la medida del casetonado de la losa prefabricada. Siendo consecuente con la idea sistémica, incluso el tamaño de los muebles respondía a dichos parámetros. En cuanto a la grilla es-

[6] El arquitecto argentino Fermín Estrella planteaba que "Ios sistemas constructivos surgen relacionados con los sistemas de proyecto" (1985: 32); en consecuencia, ante un nuevo sistema de proyecto se esperaba una respuesta constructiva inédita. Sus investigaciones sobre estas temáticas alcanzaron gran difusión en Latinoamérica en las décadas siguientes.

[7] Tal como ilustran imágenes de la maqueta presentada al concurso por el equipo que luego resultara ganador, los participantes utilizaron piezas de "Rasti" - un juego de piezas de armado por encastre, fabricado en Argentina desde la década de 1960 - a fin de ensayar alternativas para la disposición del programa arquitectónico. 

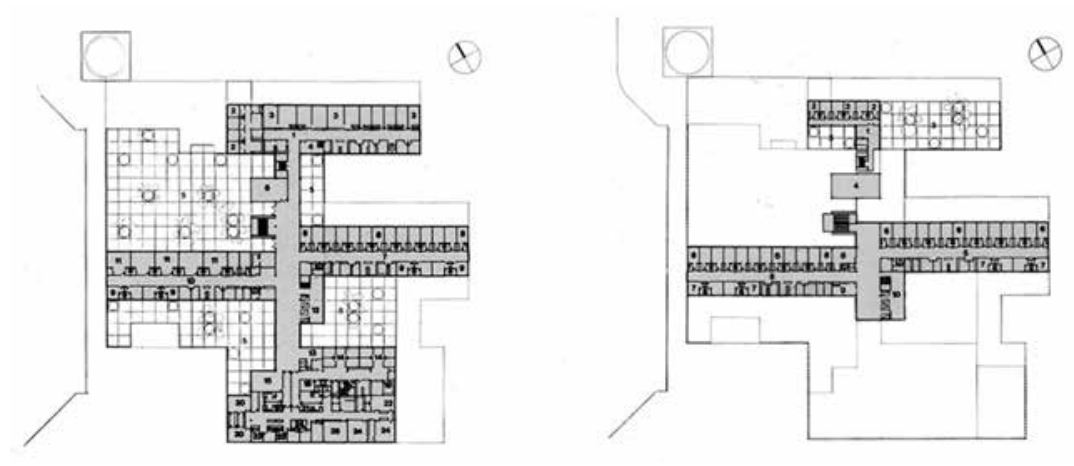

Figura 2 Llauró - Urgell. Plantas del Hospital San Vicente de Paul. Fuente: Summa, 1978, n¹29/130: 87.

tructural, la luz entre columnas fue fijada en 6,72 metros, definiendo así el "módulo básico", referencia de futuras adiciones.

Uno de los elementos más distintivos de la propuesta es la gran cubierta de sombra o doble techo de 10.000 metros cuadrados (ver Figura 3), la que guarda una deuda inocultable con los tres hospitales proyectados entre 1948 y 1951 por el arquitecto argentino Amancio Williams para la provincia de Corrientes (Müller, 2015). Ésta resuelve no solo cuestiones relativas al clima caluroso, de sol inclemente, y al riesgo sísmico del noroeste del país, sino también aspectos formales, totalizando la imagen arquitectónica al integrar el conjunto de volúmenes que, de otro modo, habría dado como resultado una volumetría fragmentaria y de difícil lectura. Los elementos que construyen el gran techo, chapas y vigas metálicas, otorgan una apariencia indisolublemente ligada a una producción industrial, factible de prefabricación y repetición. Tales decisiones llevaron a diseñar un sofisticado conjunto de elementos constructivos, expresados en dibujos que abarcaron páginas completas de las revistas especializadas del período e instalaron un imaginario técnico que proponía un horizonte deseable para la arquitectura argentina en su globalidad. Dando cuenta de su "esperanza por un mundo mejor", Juan Manuel Llauró, uno de los autores del anteproyecto ganador señaló que "la ciencia y la tecnología avanzaban cualitativa y cuantitativamente a ojos vistas, eran o nos parecían puras y sin compromisos, lo que se hiciera en base a ellos no podía dejar de encaminarse en el buen sentido" (1988: 34). En un análisis de la trayectoria del Estudio Llauró Urgell y Asociados, la arquitecta y crítica argentina Marina Waisman coincide con esta lectura, señalando que:

\footnotetext{
La preocupación por los aspectos tecnológicos de la obra arquitectónica está lejos de implicar una actitud de distanciamiento con respecto a valores humanos y sociales. No se trata de un 'tecnologismo' sino de una actitud coherente en la que la producción industrial es asumida positivamente como elemento esencial de las sociedades modernas. (1978: 43)
}

CONCLUSIONES: EL HOSPITAL DE ORÁN, UNA RESPUESTA OPTIMISTA

En 1971 el gobierno nacional da inicio a la obra, la que respeta la propuesta espacial y funcional original. Sin embargo, el contexto productivo local obligó a introducir modificaciones; finalmente, en el desarrollo del proyecto ejecutivo, una mayoría de los detalles técnicos del sistema prefabricado debieron ser reemplazados por métodos constructivos convencionales, que fueron aplicados al proyecto realizado entre 1971 y 1977. Según lo expresado por el arquitecto Augusto Penedo, quien tuvo a su cargo la adaptación del anteproyecto para los cambios impuestos por la administración pública después del golpe militar de 1976, "apenas con las estructuras de hormigón armado avanzadas en el proceso de construcción se decidió retomar la obra después de un largo período de receso y, para abaratar costos, se exigió reconvertir el edificio a técnicas tradicionales" ${ }^{\text {. }}$. Asumiendo que ya estaban contratados el hormigón estructural -que se había realizado in situ con encofrados hormigonados, en lugar de prefabricados-, la cubierta y los cerramientos, se fueron modificando los demás componentes para mantener el edificio dentro de una idea, en lo posible, coherente.

Resulta pertinente señalar que la necesidad de adecuación de las tecnologías inicialmente elegidas no solo da cuenta de las dificultades asociadas al caso analizado; más bien, deja en evidencia una coyuntura común a la práctica profesional en la Argentina del período, caracterizada por el reconocimiento de la incapacidad de la industria de la construcción local de dar respuestas con los sistemas disponibles en el mercado, por la incipiente capacitación de la mano de obra de la región, por la dificultad de conseguir financiamiento adecuado y por la lentitud de la burocracia estatal, entre otros factores. Serán, asimismo, cuestiones vinculadas con la inestabilidad del contexto socio-político argentino las que terminaron de definir el cierre de las experiencias de incorporación de sistemas industrializados a la construcción. Este desencanto también se entiende a partir 


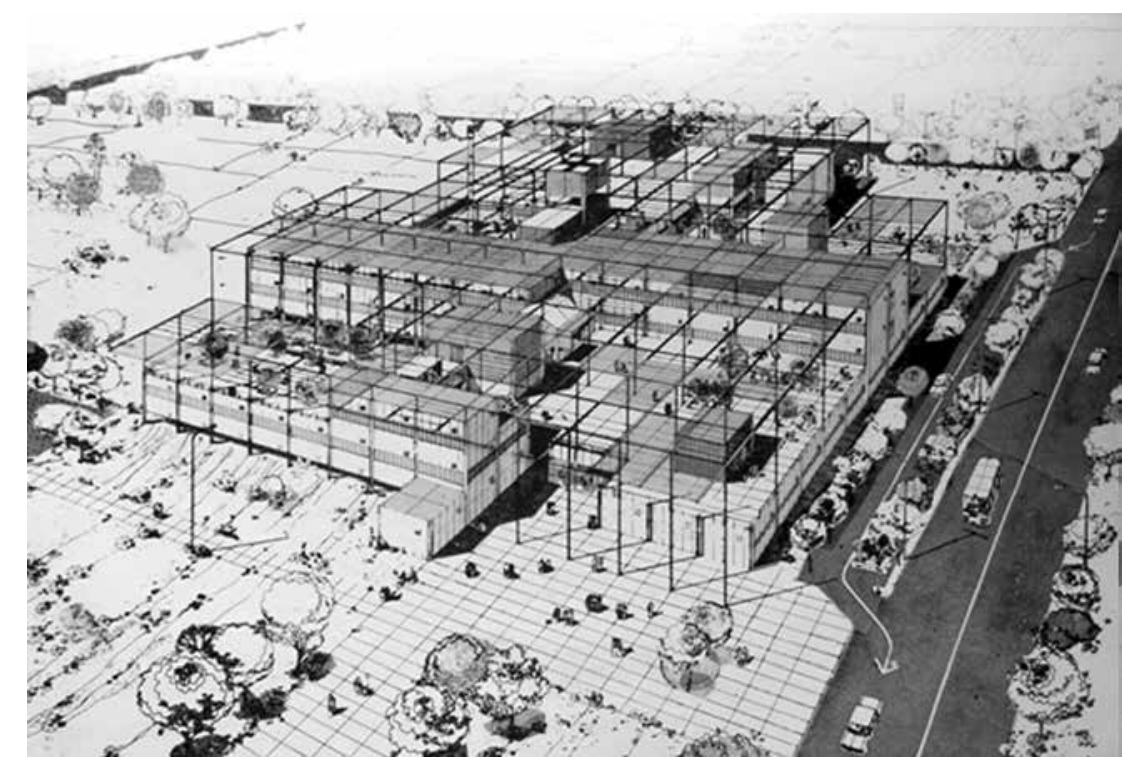

Figura 3 Llauró - Urgell. Perspectiva de concurso Hospital San Vicente de Paul. Fuente: Summa, 1978, n¹29/130: 90.
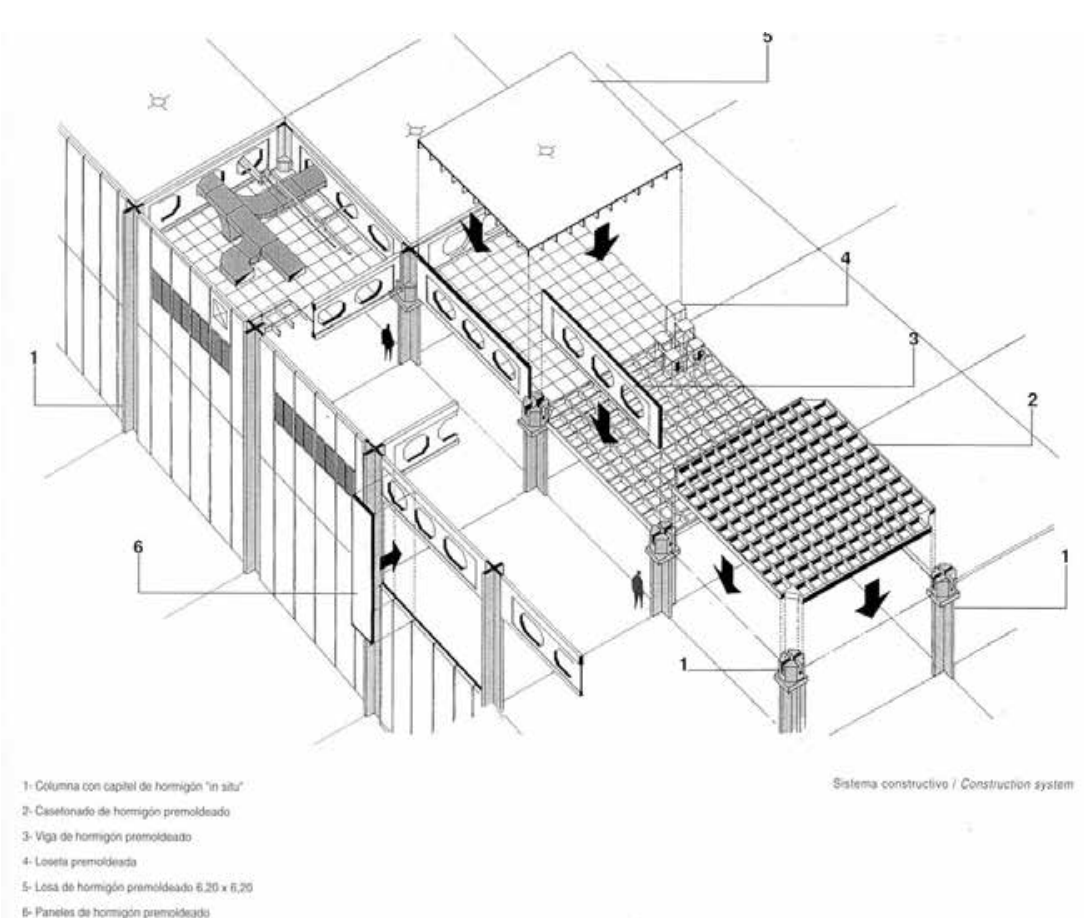

Figura 4 Llauró - Urgell. Detalle del desarrollo técnico del sistema constructivo para el concurso del Hospital San Vicente de Paul. Fuente: Summa, 1971, nº 39/40: 73. 
de reinterpretaciones contemporáneas de la idea moderna de progreso, difundidas en diversos medios como el informe "Los límites del crecimiento", elaborado por un grupo interdisciplinario de investigadores para el Club de Roma en 1972, en el que, teniendo en cuenta los niveles de incremento de la población mundial, la industrialización, la contaminación, la producción de alimentos y la explotación de los recursos naturales, se planteó que en un futuro no lejano se alcanzarían los límites absolutos de crecimiento. En la misma línea, la "crisis del petróleo" de 1973 impulsó la toma de conciencia del carácter insostenible del paradigma de consumo energético vigente, señalando el tope del crecimiento económico.

En lo que refiere a la "arquitectura de sistemas", como modalidad de proyecto que interpela el paradigma funcionalista, pocos años después de su auge las críticas comenzaron a socavar los principios sobre los que fundaba sus métodos de diseño, señalando enfáticamente que la excesiva flexibilidad no era más que una disimulada falta de definición de objetivos, que la posibilidad de crecimiento indefinido generaba grandes costos iniciales, y que la indeterminación no reconocía la existencia de áreas con funcionalidad específica y ubicación inamovible (Gentile, 1988).

Casi dos décadas después de aquel primer anteproyecto en Orán, en 1988, el arquitecto Juan Manuel Llauró, uno de los socios titulares del estudio ganador del concurso, se hacía cargo de la crítica y se preguntaba "¿qué haríamos si hubiéramos de empezar de vuelta?", y ensayaba una respuesta:

[...] coincidiríamos en que todo debía ser más modesto como aspiración de diseño. Menos paneles móviles, baños que se desplacen y salten de un lugar a otro, crecimientos y transformaciones ilimitadas. Menos sueños de alcanzar un edificio que controle el futuro, los cambios y alteraciones del devenir; la situación en nuestro país en la actualidad demuestra que el futuro no se compra, no se lo domina. (Llauró, 1988: 35)

Su reflexión muestra, de igual modo, su compromiso social, ya que para el estudio profesional la concreción del hospital "significaba romper una situación injusta y proyectarnos hacia un futuro mejor" (ibídem: 34). La cita es elocuente respecto al clima de confianza que numerosos profesionales tenían por entonces en la arquitectura como protagonista activa de la renovación disciplinar, encontrando diversas vías para expresar el cambio de rumbo (Müller y Parera, 2015). La arquitectura de sistemas será [precisamente] una de ellas, caracterizada por la firme creencia en el progreso en manos de la técnica, a la que consideraban neutral (Silvestri, 2014) y carente de fines retóricos (Aliata, 2004).

La arquitectura es una de las pocas profesiones que mira al futuro haciéndose cargo de su pasado. En ese sentido, tiene el deber de ser optimista pero, a la vez, la necesidad de saber leer las coordenadas que sitúan su producción en un tiempo y un lugar determinados. La "arquitectura de sistemas" resultó ser un gran aporte a la solución de programas complejos y de gran importancia en su uso social, aspectos que la mayoría de las veces se resolvieron con eficacia, contribuyendo a dar flexibilidad organizativa y adaptabilidad a los edificios. En la misma línea, la multiplicación de concursos como modos de selección de proyectos generó un momento propicio para el reconocimiento de jóvenes profesionales y la difusión de propuestas innovadoras, como las presentadas a la convocatoria del Ministerio de Bienestar Social en 1969. Pero en algunos casos, como el estudiado, este enfoque sistémico pudo haberse excedido en su optimismo, generando sus propios límites o, mejor dicho, proponiéndose metas constructivas inalcanzables bajo las particulares circunstancias que lo rodeaban.
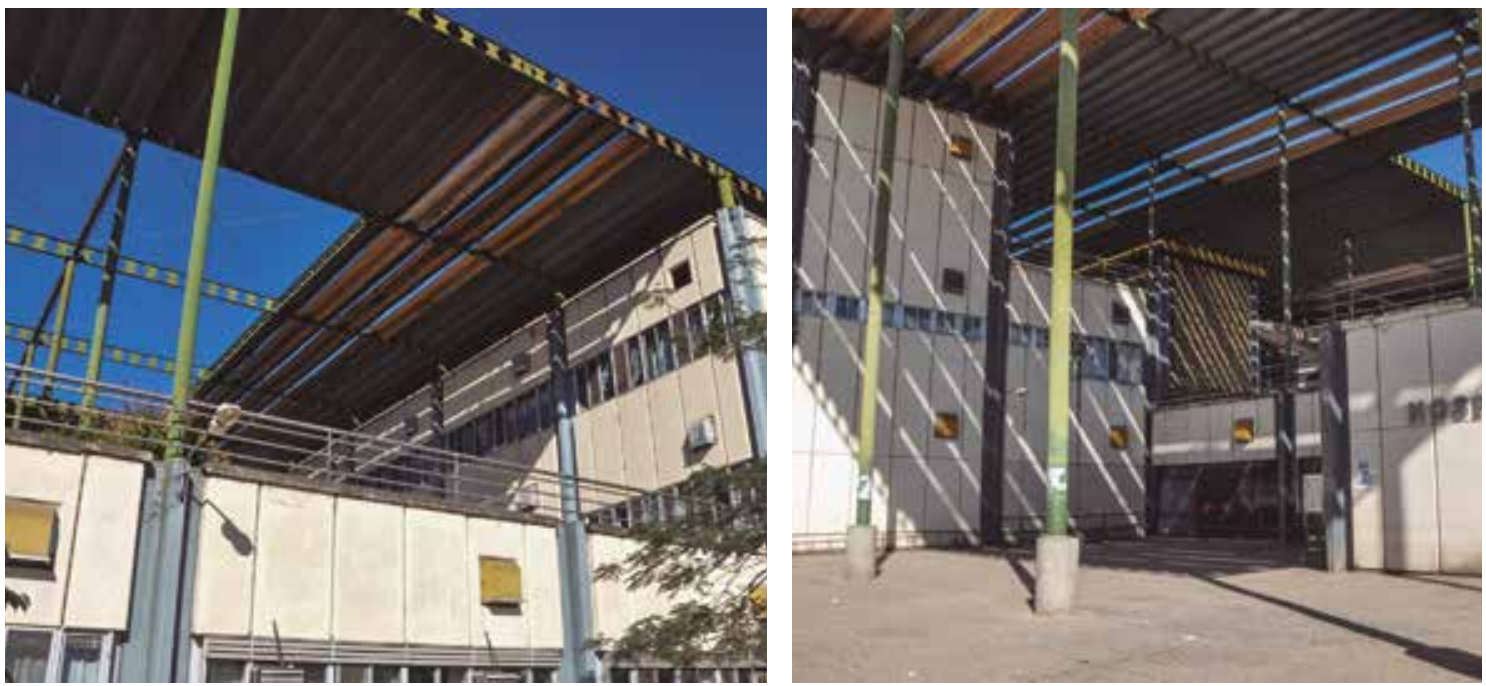

Figura 5 Llauró - Urgell. Hospital San Vicente de Paul. Fuente: Fotografías del arquitecto Rubén Cabrera, 2011. 


\section{REFERENCIAS BIBLIOGRÁFICAS}

ALIATA, Fernando. Sistemas, arquitectura de. En: LIERNUR, Francisco y ALIATA, Fernando. Diccionario de Arquitectura en Argentina. Buenos Aires: AGEA, 2004, pp. 57-60.

ALIATA, Fernando. Arqueología de la arquitectura de sistemas. Registros, julio 2014, año 10, n 11, pp. 6-19.

BULLRICH, Francisco. Arquitectura argentina 1960/70. Summa, octubre 1969, n 19, pp. 37-82.

ESTRELLA, Fermín. Arquitectura de sistemas al servicio de las necesidades populares, 1964-1983. Buenos Aires: Hachette, 1985.

FRIGERIO, Rogelio. Crecimiento económico y democracia. Buenos Aires: Losada, 1963.

GANDELSONAS, Mario. Un enfoque teórico de la arquitectura. Summa, diciembre 1970, n 32, pp. 69- 73.

GENTILE, Patricia. Concursos de hospitales del 70, veinte años después. Trama, 1988, n²1, pp. 32-33.

LIERNUR, Jorge F. Arquitectura en la Argentina del Siglo $X X$. La construcción de la modernidad. Buenos Aires: Fondo Nacional de las Artes, 2001.

LLAURÓ, Juan Manuel. Orán (Hospital San Vicente de Paul - San Ramón de la Nueva Orán). Trama, 1988, n²1, pp. 34-35.

MINISTERIO DE BIENESTAR SOCIAL. Pautas de diseño en arquitectura hospitalaria. Summa, julio/agosto 1971, n $39 / 40$, p. 30

MONTAGÚ, Arturo. Método sistemático de diseño. Su relación con el diseño arquitectónico. Revista de la SCA $1972, n^{\circ} 69$, pp. 27-31.

MOREA, Luis Miguel; STAGNI, Pablo y HUMAR, Raúl. La vivienda industrializada. Summa, noviembre $1973, \mathrm{n}^{\circ} 69$, pp. 18-24.

MÜLLER, Luis. Invenciones, sistemas y tecno utopías. De los hospitales correntinos de Williams al hospital en Orán de Llauró - Urgell. Documento presentado en XIV Jornadas de investigación INTHUAR. FADU UNL, Santa Fe, junio 2015.

MÜLLER, Luis y PARERA, Cecilia. Identificación programática, texturas rugosas. Alternativas críticas al interior de la arquitectura moderna en Argentina. Documento presentado en I Congreso Nacional e Internacional de Historia del Arte, Cultura y Sociedad, Universidad Nacional de Cuyo. Mendoza, octubre 2015

PREBISCH, Raúl. Problemas teóricos y prácticos del crecimiento económico. México: CEPAL, 1952.

RAPOPORT, Mario. Historia económica, política y social de la Argentina (1880-2003). Buenos Aires: Emecé, 2005.

RIVAROLA, Raúl. Hospitales. Summa, julio/agosto 1971 $n^{\circ} 39 / 40$, p. 29.
SCHERE, Rolando. Concursos 1885-2006. Buenos Aires: SCA, 2008

SILVESTRI, Graciela. Alma de arquitecto. Conformación histórica del "habitus" de los proyectistas del hábitat. Registros, julio 2014, año 10, n 11, pp. 72-97.

SHMIDT, Claudia y MÜLLER, Luis (comps.). La "Teoría de sistemas" en la transformación de la cultura urbana. Arquitectura, ciudad y territorio entre el profesionalismo y la tecno-utopía (1950-1980). Buenos Aires: UTDT, 2013.

SUBSECRETARÍA DE SALUD PÚBLICA. Programa Nacional de Planeamiento Físico y Arquitectura. Summa, octubre 1971, n 41 , pp. 29-44.

VON BERTALANFFY, Ludwig. General System theory: Foundations, Development, Applications. Edmonton: University of Alberta, 1968

WAISMAN, Marina. Reflexiones sobre la obra de LlauróUrgell y Asociados. Summa, octubre/noviembre 1978, n 129/130, pp. $42-43$. 